\title{
„Handle, wie Dir Dein Dämon vorschreibt!““
}

\section{Die illusionistische Weltanschauung Oskar Panizzas und ihre Aporien}

Im Aufsatz wird die Rede sein sowohl vom turbulenten Leben des deutschen Schriftstellers, Essayisten und Satirikers Oskar Panizza (1853-1921) als auch von seinem Konflikt mit dem Gesetz und der Obrigkeit, weshalb er ein Jahr im Gefängnis verbringen musste. Auf der Grenze zwischen Realität und Illusion, Genie und Wahnsinn balancierend, hat dieser charismatische Autor ein Leben voller Widersprüche durchlebt: Er hat als Arzt für mentale Krankheiten begonnen, wurde dann aber Schriftsteller, um seine Lebensodyssee in einem Sanatorium zu beenden. Im Anschluss an Max Stirner hat Panizza den Illusionismus als berechtigte Weltanschauung vertreten: Halluzination ist das eigentlich Reale, die Realität bloß eingebildet. Im Aufsatz soll aber gezeigt werden, dass es sich nicht um einen geistig umnebelten kreativen Kopf handelt, sondern um jemanden, der die Grenze zwischen dem Normalen und Abnormalen hinterfragt hat, womit er im bestimmten Sinne die kommende Kritik der normativen Voraussetzungen der institutionalisierten Psychiatrie vorweggenommen hat.

\section{Schlüsselwörter}

Oskar Panizza, Illusionismus, Dämonismus, Individualismus, Genie, Wahnsinn, Psichopatia criminalis
\end{abstract}

Die Welt ist ein Gefängnis, in dem Einzelhaft vorzuziehen ist.

Karl Kraus

\section{Oskar Panizza: ein Grenzgänger zwischen Genie und Wahnsinn}

In der Alltagssprache hat sich die Rede vom Grenzgang zwischen Genie und Wahnsinn eingebürgert. Diese Redeweise wird vor allem auf jene schöpferischen Naturen angewendet, die oft am Höhepunkt ihres Schaffens die Grenze des psychisch Gesunden überschritten haben und manchmal auch dem Wahnsinn verfallen sind. Man hat infolge der Entwicklung der Psychologie als wissenschaftlicher Disziplin mit bestimmten normativen Implikationen ${ }^{1}$ versucht, die Trennlinie zwischen genialer Schöpferkraft und psychischer Erkrankung aufzuheben, was ziemlich problematisch ist. ${ }^{2}$ Die Pathologisierung des Schöpferischen mag daher gerade als eine Krise des Schöpferischen gedeutet werden - die Apologeten des Durchschnitts verdammen alles was über

1

Im Sinne dessen, was gesund (normal) und was krank (abnormal) ist.
2

Man denke etwa an die angeblich ,wissenschaftlich" fundierten Versuche einer Aufhe- 
oder unter ihm zu liegen scheint. Während man früher die schöpferische Gabe eines Künstlers sogar als Ergebnis der göttlichen Eingebung aufgefasst hat, werden an der Schwelle zur Moderne den Genialen Zwangsjacken auferlegt. Nach der Phase der romantischen Verklärung der Genialität kam die Phase der realistischen Ernüchterung, infolge welcher jene gleich einer Geisteskrankheit als Abweichung vom Normalen gedeutet wurde. Die Dialektik von Genie und Wahnsinn in der Regie der psychiatrisch disziplinierenden Vernunft soll uns aber hier nicht interessieren, so inspirierend (auch aus bioethischer Sicht) ihre Interpretation für die Aufdeckung des tieferen Zusammenhangs von Macht, Wissens- und Subjektproduktion im modernen Zeitalter wäre, wie sie etwa der Diskursanalytiker Michel Foucault in seinen Schriften vorangetrieben hat.

Die Aufgabe des vorliegenden Aufsatzes ist wesentlich bescheidener. Es soll an einem einzelnen Fall aufgezeigt werden, wie manchmal die Grenze zwischen künstlerischer Schöpfungskraft und psychischer Erschöpfung porös wird - oder wie ihre Aufhebung gesellschaftlich inszeniert werden kann, wenn es darum geht, Außenseiter zu disziplinieren. Ein Fall für Foucault dürfte auch der des deutschen Schriftstellers Oskar Panizza (1853-1921) gewesen sein, des Skandalautors am Ausgang des 19. Jahrhunderts, der auch eine Haftstrafe verbüßen musste, um im letzten Viertel seines bewegten Lebens nach realen und bloß eingebildeten Verfolgungen, abgeschottet vom äußeren Geschehen in einem Sanatorium, allmählich in geistiger Umnachtung zu enden. Was den „Fall Panizza“ umso interessanter macht, ist dass er selbst zum Psychiater ausgebildet wurde und eine gewisse Zeit auch als solcher tätig war - aber am Ende selber zum psychiatrischen Fall geworden ist. Was Panizzas Lebens- und Leidensgeschichte in philosophischer Hinsicht relevant macht, ist seiner Hinterfragung der erkenntnistheoretischen Grunddifferenz zwischen Realität und Illusion zu verdanken, welche er in seiner Schrift Der Illusionismus und die Rettung der Persönlichkeit (1895) unter dem Einfluss des radikalen Individualisten Max Stirner vorgenommen hat. Außerdem hat er - um noch einmal die bereits erwähnte Unterscheidung zu erwähnen - die Grenze zwischen Genie und Wahnsinn ebenfalls problematisiert und theoretisch (in einem Vortrag über dieses Thema) bzw. praktisch (durch sein eigenes Leben) hinterfragt.

Nachdem zunächst einzelne Stationen des umtriebigen Lebens des Skandalautors kurz geschildert werden, soll seine illusionistische Weltanschauung vorgestellt und die Frage ihrer philosophischen Relevanz gestellt werden. Das Ganze soll jedoch mit Fragen abgerundet werden, in denen Panizzas Krankheit und die Art, wie er mit ihr umgegangen ist, thematisiert wird, mit der Konsequenz, dass der Begriff der Einbildung wieder ins Zentrum rückt - und die entscheidende Frage gestellt wird, ob diesem Begriff auch etwas Positives abgewonnen werden könnte, was möglicherweise Panizza in seiner Abhandlung über den Illusionismus zum Ausdruck bringen wollte.

\section{Aus dem Leben eines umstrittenen Autors ${ }^{3}$}

Oskar Panizza wird am 12. November 1853 im fränkischen Bad Kissingen geboren. Der kleine Oskar genießt eine strenge religiöse Erziehung. Seine schulische Ausbildungszeit verläuft ziemlich turbulent. Nach schlechten Leistungen verlässt er 1872 ein Münchener Gymnasium und besucht eine Handelsschule - ebenfalls ohne Erfolg. In dieser Zeit nimmt er auch Gesangs- 
unterricht im Münchener Konservatorium. Der Versuch kurze Zeit darauf bei einem Nürnberger Bankhaus, die Lehre zu absolvieren, schlägt fehl. Nach einem einjährigen freiwilligen Militärdienst und der Genesung von Cholera schreibt er sich 1876 an einem Schweinfurter Gymnasium ein und beendet das Abitur mit herausragenden Noten. Im selben Jahr schreibt er sich für das Medizinstudium in München ein, das er 1880 mit der Promotion zum Doktor der Medizin abschließt. Bereits vor dem Ende des Studiums reist er nach Italien, wo er sich vermutlich mit Syphilis infiziert. ${ }^{4}$ Nach dem Studium hält er sich in anderen Ländern auf (England, Frankreich). Die Mobilität bleibt das Kennzeichen seines unruhigen und rastlosen Lebens. Nachdem er eine Zeit lang als Militärarzt und dann als Assistenzarzt in einer psychiatrischen Anstalt gearbeitet hat, gibt er diesen Beruf auf Grund von Unstimmigkeiten mit seinen Vorgesetzten und gesundheitlichen Problemen auf. Ab 1884 wendet er sich der Literatur zu, nachdem er finanziell durch eine Rente abgesichert war. 1885 beginnt das literarische Talent Panizzas seine ersten Früchte zu tragen. Es erscheint die Gedichtsammlung Düstre Lieder, ein Jahr später die Londoner Lieder (entstanden während seines erneuten Aufenthalts in der englischen Metropole). 1890 beginnt er Beziehungen zu den Mitgliedern des Münchener Literatenkreises zu knüpfen, welcher sich um den charismatischen Schriftsteller Michael Georg Conrad gebildet hat. Im selben Jahr erscheint unter dem Einfluss von E. T. A. Hoffmans und E. A. Poes Schauererzählungen der erste Erzählband Dämmerungsstücke. Nebenbei beginnt er sich auch als Journalist zu betätigen. 1891 wird er beinahe wegen seiner provokanten Erzählung „Das Verbrechen in Tavistock-Square“ angeklagt. Es folgen die satirische Erzählung „Aus dem Tagebuch eines Hundes“ (1892), der Erzählband Visionen (1893) und die anonym erschienene Schrift Die unbefleckte Empfängnis der Päpste, welche 1893 verboten und beschlagnahmt worden ist. Dasselbe Schicksal ereilt sein Drama Das Liebeskonzil. Eine Himmelstragödie in fünf Aufzügen (1895). Doch es bleibt nicht dabei - Panizza muss sich vor dem Münchener Landgericht wegen des Vorwurfs der Gotteslästerung verantworten. In seiner Verteidigungsrede, ${ }^{5}$ welche als leidenschaftliches Dokument der Verteidigung des Rechts des Intellektuellen auf freie Meinungsäußerung

bung der Grenze zwischen Genie und Irrsinn bei dem italienischen Arzt Cesare Lombroso, die letztlich durch verschiedene Ideologien (wie z. B. die nationalsozialistische) missbraucht worden sind.

\section{3}

Vgl. dazu seine eigene Skizze: Oskar Panizza, „Selbstbiographie“, in: Oskar Panizza, Psichopatia criminalis und andere Schriften, Vollständige Neuausgabe mit einer Biographie des Autors, herausgegeben von Karl-Maria Guth, Hofenberg, Berlin 2015, S. 151-159. Vgl. zu den biografischen Daten auch ebd., S. 160-164.

4

Das Thema ,Syphilis‘ beschäftigt ihn auch später - in seinem ketzerischen Drama Das Liebeskonzil nimmt er die vermutete Zeit des Ausbruchs dieser tückischen Geschlechtskrankheit in Italien am Ende des 15. Jahrhunderts zum Anlass für eine bitterböse Abrech- nung mit der römischen Kirche. Die Epidemie wird als Folge eines „Deals“ zwischen Gott und Teufel interpretiert, damit die Vertreter der kirchlichen Macht für ihr ausschweifendes Leben und den eigentlichen Ungehorsam gegenüber dem Herrn bestraft werden. Der Teufel verlangt als Gegenleistung für seine Hilfe nur eine Reparatur des Eingangs zur Hölle und die Freiheit des Denkens. Man merkt dieser Forderung den Sarkasmus Panizza'scher Prägung an. (Vgl. Oskar Panizza, Das Liebeskonzil, Mit der Verteidigung des Werkes vor dem Landgericht München, Neuausgabe mit einer Biographie des Autors, herausgegeben von Karl-Maria Guth, Hofenberg, Berlin 2017.)

Sie wird 1897 als selbstständige Schrift publiziert. Vgl. Oskar Panizza, ,Meine Verteidigung in Sachen ,Das Liebeskonzil'“", in: O. Panizza, Psichopatia criminalis und andere Schriften, S. 4-27. 
noch immer zu fesseln vermag, versucht er den Sinn und die Berechtigung der satirischen Religionskritik aufzuzeigen. Auch wenn sich viele Intellektuelle - zu dieser Zeit und danach - für Panizza ausgesprochen haben (M. G. Conrad, Theodor Lessing, Karl Kraus), hat ihm das nicht viel geholfen. Nachdem unter den Geschworenen viele halbgebildete Menschen waren, die Panizzas Stück vermutlich gar nicht gelesen (geschweige denn: verstanden) haben, braucht man sich nicht zu wundern, dass der Provokateur diesmal der Strafe nicht entkommen konnte. So wurde Panizza zur einjährigen Haftstrafe in Amberg verurteilt. 1895 erscheint seine unter dem Einfluss Max Stirners entstandene philosophische Schrift Der Illusionismus und die Rettung der Persönlichkeit. Skizze einer Weltanschauung. Nach dem Ende der Gefängnisstrafe kehrt Panizza der bayrischen Hauptstadt buchstäblich den Rücken zu und schreibt seinen Abschied von München - auch dieses Werk wird verboten und Panizza muss ins Ausland fliehen. Damit beginnt die Agonie der letzten Jahre vor seinem geistigen Zusammenbruch.

In seinem Schweizer Exil gründet er einen eigenen Verlag und eine eigene Zeitschrift (Zürcher Diskußjonen). 1898 veröffentlicht er seine satirische Abhandlung Psichopatia criminalis, in der er den Hütern des Gesetzes und der seelischen Gesundheit Ratschläge zur Behandlung einer imaginierten Krankheit gibt, welche jene Subjekte befällt, welche sich gegen die Autorität des Staats und der Kirche auflehnen. Im November dieses Jahres muss er wegen des Verhältnisses zu einer minderjährigen Prostituierten ${ }^{6}$ erneut die Flucht ergreifen und eine neue Wahlheimat suchen - diese findet er in Frankreich. 1899 veröffentlicht er dort die Gedichtsammlung Parisjana. Deutsche Verse aus Paris, in der er das politische Regime des Kaiserreichs anprangert, woraufhin eine internationale Fahndung nach ihm eingeleitet wird. Ein Jahr später wird sein Vermögen durch die bayrischen Behörden beschlagnahmt, sodass er fast ohne Geldmittel bleibt. Allmählich beginnt sich sein psychischer $\mathrm{Zu}$ stand zu verschlechtern - er leidet zunehmend unter Halluzinationen. Völlig ohne Mittel geblieben, bleibt ihm nichts anderes übrig als sich den Behörden zu übergeben. Er macht dies im April 1901 und wird schließlich verhaftet. Im August dieses Jahres wird er aus der Irrenanstalt entlassen, in die er wegen der Diagnostizierung der Art und des Grads seiner psychischen Gesundheit eingewiesen worden ist (man hielt ihn nicht für zurechnungsfähig - deshalb konnte man ihn nicht wegen seiner ketzerischen Gedichte verurteilen). Panizza kehrt wieder nach Paris zurück, kann aber dem inneren psychischen Druck nicht mehr standhalten und bildet sich ein, von den Handlangern des Kaisers Wilhelm II. verfolgt zu werden. Bis April 1904 arbeitet er an Imperjalja, an einer Schrift, in der alle Verbrechen des Kaisers genannt werden sollten. Diese Abrechnung mit dem kaiserlichen Regime wird jedoch nicht zu seinen Lebzeiten veröffentlicht.

Nachdem er wieder in der Heimat angekommen ist, versucht Panizza seine Internierung in einer Heilanstalt buchstäblich zu provozieren - er läuft am 19. Oktober 1904 halbnackt durch die Münchener Straßen und wird daraufhin im Februar 1905 in eine Bayreuther Anstalt eingewiesen. Zwei Monate später wird er wider Willen entmündigt. Schließlich wird er im März 1908 in das Sanatorium Herzogshöhe nahe bei Bayreuth gebracht, wo er die letzten Jahre seines unruhigen Lebens verbringt. Er stirbt an den Folgen eines Schlaganfalls am 28. September 1921 und wird auf dem Bayreuther Friedhof begraben. Angeblich ohne Grabinschrift - was wohl die Maßnahme seiner Verwandten war, welche keine Solidarität mit dem rebellischen Verstorbenen zeigen und 
jegliche Erinnerung an ihn vertilgen wollten. Aber vergebens! Der Nachruhm Oskar Panizzas konnte nicht aufgehalten werden, trotz - oder gerade wegen - aller Verleumdungen seiner Persönlichkeit und seines literarischen Werks.

\section{Panizzas radikaler Individualismus}

Wie bereits ein flüchtiger Blick auf sein bewegtes Leben zeigt, handelt es sich im Falle von Oskar Panizza um einen eigensinnigen Außenseiter, der den aussichtslosen „Sisyphoskampf“ gegen die staatlichen Autoritäten und verlogene Doppelmoral seiner Zeit aufgenommen hat - um am Schluss den Kürzeren zu ziehen. Jemand, der seinem Dämon vertraut hat und an ihm zu Grunde gegangen ist. Aber nicht nur in seinen Stellungnahmen wie in der Verteidigungsrede oder seinem leidenschaftlichen „Abschied aus München“ hat der fränkische Schriftsteller seine Radikalität unter Beweis gestellt - auch in seinen eher theoretischen Texten fällt die Signatur seines „,inneren Dämons“ auf. Seine philosophische Abhandlung Der Illusionismus und die Rettung der Persönlichkeit (1895), welche er kurz vor dem Antritt seiner Haftstrafe in Amberg verfasst hat, trägt das Gepräge eines unruhigen Freigeistes, der eine Befreiung der Persönlichkeit aus den Fangen eines materialistischen und konservativen Weltbilds angestrebt hat.

Bereits die Widmung am Anfang der Schrift zeigt die Richtung, in der sich seine Argumentation (aber auch Apellation an die Leser) bewegen wird: Max Stirner (1806-1856) ist die Figur, von der sich Panizza zu seinem radikal individualistischen Ansatz inspirieren ließ. In doppelter Hinsicht ließ er sich von diesem „Lazarus unter den Filosofen ${ }^{768}$ zu seinen eigenen Ideen anspornen: einmal in inhaltlicher Hinsicht, da sich bei Stirner eine Auffassung des Denkens auffinden lasse, welcher der gängigen empirisch-wissenschaftlichen Sicht widerspricht, dann aber auch in stilistischer Hinsicht, denn in der „Leichtigkeit und Flüssigkeit des Vortrags“, wie ihn Stirner pflegt, könne das Thema wesentlich griffiger präsentiert werden als im gängigen Stil der akademischen Philosophie (Panizza spricht spöttisch vom ,zähen Asfalt-Brei aus dem Munde patentirter Sanskritisten“). ${ }^{9}$ Stirner setzte sich zur Aufgabe, eine ganze Reihe von Hirngespinsten zu entlarven, welche seiner Ansicht nach den Weg zum „Ich“ des Einzigen versperren, so als ob der alleinige Sinn dieser Welt in ihnen läge. Solche Chimären sind Gott, Geist, Mensch, welche

Es waren vermutlich eher politische Gründe, die ihn zum Verlassen der Schweiz bewegt haben.

7

Man lasse sich nicht von der Schreibweise irritieren, welche man in Panizzas Schriften antrifft. Seine radikale Individualität spiegelt sich auch in der Weise wider, wie er geschrieben hat, damit auch sein Widerstand gegen gängige Konformitätsmuster, welche sich selbst in der Rechtsschreibung niederschlagen. Panizza hat die Wörter der deutschen Sprache so geschrieben, wie sie ausgesprochen werden. Also beispielsweise ,Filosofen', ,Materjalismus', ,Glük', ,Halluzinazion', ,Schurnalist' statt ,Philosophen', ,Materialismus', ,Glück', ,Halluzination',
,Journalist‘. Vgl. zu dieser orthografischen Besonderheit Panizzas Jürgen Müller, Oskar Panizza - Versuch einer immanenten Interpretation, Inaugural-Dissertation zur Erlangung der Doktorwürde der Medizinischen Fakultät der Bayerischen Julius-Maximilians-Universität zu Würzburg, Würzburg 1990, S. 46, Fußnote 121.

8

Oskar Panizza, „Der Illusionismus und die Rettung der Persönlichkeit“, in: O. Panizza, Psichopatia criminalis und andere Schriften, S. 28-73, S. 29.

Vgl. ebd. 
dann in noch höheren Abstraktheiten wie Kirche, Gemeinschaft, Staat ,aufgehoben“werden. Demgegenüber setzt der Egoist im Stirner'schen Sinne die Karte auf sich selbst, sein Ich, will die ganze Welt „enteignen“ und sich nur um seine eigene Sache kümmern. Der Egoismus Stirner'scher Prägung will alle „Ich-unterdrückenden“ Weltanschauungen und Ideologien - christliche Religion, Idealismus, Anthropozentrismus, Liberalismus, Kommunismus o. dgl. - hinter sich lassen und allein das Ich des Einzigen und dessen Eigentum zu seinem Recht kommen lassen. Diese extreme Ansicht provozierte Widerstand bereits zu Stirners Lebzeiten wie die Replik seiner Zeitgenossen, Ludwig Feuerbach oder Bruno Bauer, ferner die scharfe Kritik von Marx und Engels in Der deutschen Ideologie zeigt, was zu einem negativen Bild des radikalen Denkers in der Philosophie beigetragen hat. Panizza sieht dagegen in ihm ein Vorbild für sein ketzerisches Denken und trägt mit seiner Interpretation wie mit seiner Fortsetzung der Stirner'schen Denkart zu einem anderen Bild Stirners bei, das sich eher in die Reihe der freigeistigen Denker wie Friedrich Nietzsche fügt.

Es gab also auch einen ,äußeren“ Dämon und nicht nur einen ,inneren“, der Panizza zu seinen Gedanken angespornt hat. Ähnlich gesellt sich zu ähnlich, wie man so sagt, und das trifft auch hier zu: Auf einen Skandaldenker folgt der andere, eine Provokation kommt nach der nächsten, Panizzas „Dämon“ tritt die Nachfolge des „Einzigen“ an. Radikaler Individualismus hie, noch radikalerer dort - bei der Lektüre sowohl von Panizzas Schrift wie von Stirners Hauptwerk werden wir nachträglich Zeugen einer Vergötterung des Individuums, welche man in dieser Form nur noch bei Friedrich Nietzsche antreffen kann. Was hat es mit dieser radikalen Aufwertung des Individuums auf sich?

Ausgangspunkt für Panizzas Überlegungen ist die geistige Situation seiner Zeit - die Ablöse der idealistischen Systeme durch den naturwissenschaftlichen Materialismus. Infolge dieses Weltanschauungswechsels verkommt das Denken zu einem Korrelat der Gehirntätigkeit, es lässt sich - vor allem in der aufkommenden experimentellen Psychologie - quantifizieren und anhand von messbaren Daten identifizieren. Umso überraschender kommt Panizzas Absage an diese Art der Abwertung der Eigenständigkeit des Denkens, denn man würde von jemandem, der selbst die auf naturwissenschaftlicher Basis fundierte Ausbildung zum Psychiater absolviert hat, erwarten, dass er vom damals populären psychophysischen Parallelismus angeregt, die skeptischen und reduktionistischen Vorstellungen seiner akademischen Zeitgenossen teilt, wenn es um die Selbstständigkeit der Seele und des Denkens geht. Aber Panizza liebte es anscheinend überall gegen den Strom zu schwimmen, nicht nur im alltäglichen Leben, sondern auch auf theoretischer Ebene. Geht man von der Radikalität seiner Persönlichkeit aus, so lässt sich auch seine Aversion gegenüber dem damaligen intellektuellen Mainstream erklären. ${ }^{10}$

Was ist das größte Problem gewesen, welches den Vertretern der verschiedenen Spielarten des Materialismus - sowohl zu Panizzas als auch zu früheren Zeiten - Kopfzerbrechen bereitet hat? ${ }^{11}$

„[D]as, was die Materjalisten am liebsten los wären, und am liebsten los sein wollen müssen, ist - das Denken." ${ }^{\text {"12 }}$

Auf der anderen Seite haben diejenigen, die sich nicht dem reduktionistischen Weltbild der Materialisten verschrieben haben, einen Rettungsanker, der dem in eine Krise geratenen menschlichen Geist helfen könnte: 
„Für Einen, dem die Vorstellung, dass sein gesamtes Dasein als Mechanismus beschlossen sei, unerträglich wäre, was bliebe ihm übrig? - Sich in sein Denken zu retten." ${ }^{\text {"13 }}$

Also muss vom Denken ausgegangen werden. Wenn man sich auf den Pfad des persönlichen (seines eigenen) Denkens einlässt, was erwartet da Einen?

„Was ist (...) dasjenige persönliche Erlebnis in uns, welches uns am entschiedensten, am direktesten, oft in erschreckender Weise, den Gedanken von der Genuität, von der Ursprünglichkeit des Denkens nahelegt? - Der Zwangs-Gedanke. Die Inspirazion. Die Halluzinazion. " ${ }^{14}$

Es mag überraschend sein, dass Panizza als Hauptformen des persönlichen Denkerlebens eigentlich deviante und fast pathologische Formen nennt. Aber es sind gerade die nicht zu kontrollierenden Einfälle und Vorfälle des Denkens, welche den prozesshaften, ja ereignishaften Charakter des Denkens gut repräsentieren, gewissermaßen seine Selbstständigkeit und Widerspenstigkeit gegenüber materialistischen Reduktionen. Dieses geistige Erlebnis, das also ein Denkereignis ist, wird von Panizza als ,direkter Hohn auf alle kausale Verknüpfung " ${ }^{\text {"15 }}$ bezeichnet. ${ }^{16}$

Er möchte es vermeiden, solche Denkprozesse aus einem „Unterbewusstsein“ oder gar „Unbewussten“ heraus zu erklären und insistiert auf der Unwiederholbarkeit und Singularität solcher mentalen Ereignisse. Sie tragen buchstäblich das Siegel der Individualität der Person, deren Denken in Frage steht. Um möglichen Auseinandersetzungen mit der traditionellen Philosophie (vor allem mit Kants transzendentaler Philosophie) bewusst aus dem Weg zu gehen, wählt der Individualist Panizza den Ausdruck ,Dämon ${ }^{17}$ (sic!), um das fragliche metaphysische Prinzip, das sich „hinter“ oder „unter“ dem Denken des Einzelnen befindet, auf einen Nenner zu bringen. Und zwar aus dreierlei Gründen: Erstens, weil damit die Qualitäten dieses Prinzips treffend formuliert werden (es ist schaffend, wirksam, eingebend, vordrängend etc.); zweitens, weil es eine Reminiszenz an Sokrates ist; drittens, weil damit individuelle (,meine“) Eingebungen gedeutet werden sollen, das, was ein einzelner

10

Möglicherweise haben zu seiner Kritik der szientistischen Weltsicht auch seine eigenen Erfahrungen als Student der Medizin und später als Militärarzt beigetragen.

11

Und davon sollte man nicht die heutigen ,eliminativen" und sonstigen Materialisten ausnehmen.

12

O. Panizza, ,Der Illusionismus und die Rettung der Persönlichkeit", S. 35.

13

Ebd.

14

Ebd., S. 36.

15

Vgl. ebd., S. 37.

16

Panizza lehnt die Annahme von ,unbewussten Vorstellungen“ ab, um auf diese Weise
Zwangsgedanken und Einfälle ,wegzuinterpretieren". Vgl. zu seiner Kritik, die sich auch gegen die Idee einer „Philosophie des Unbewussten" richtet, ebd., S. 37 f., vor allem Fußnote 2. Er nennt zwar nicht seinen Namen, aber er meint wohl damit auch Eduard von Hartmanns Hauptwerk, welches Ende des 19. Jahrhunderts geradezu bestsellermäßigen Erfolg hatte. Vgl. Eduard von Hartmann, Philosophie des Unbewussten, Vollständiger Text der zweiten Auflage, Neuausgabe, herausgegeben von Karl-Maria Guth, Hofenberg, Berlin 2017.

17

Er findet dazu ein Analogon in der altindischen Philosophie, wo die Rede vom Brahman ist. Vgl. O. Panizza, „Der Illusionismus und die Rettung der Persönlichkeit“, S. 44, Anm. 3. 
Mensch beim Denken erlebt und nicht die Mehrheit der Menschen. Das Dämonische wird entmystifiziert und philosophisch ausgedeutet:

„Der Dämon ist (...) ein aus dem Transzendentalen mit Notwendigkeit gewonnener Faktor, um mein mit Kausalbedürfnis ausgestattetes diesseitiges Denken und die an ihm hängende Erscheinungswelt zu erklären." ${ }^{18}$

Es gibt kein „Ding an sich“ - nur Erscheinungen. Aber diese Erscheinungen sind selber nur Illusionen, hinter denen der (persönliche) Dämon steht. Er ist eigentlich das „An sich“, welches nach dem Abzug der Sinnestätigkeit übrigbleibt. Jeder Mensch lebt, angetrieben von seinem persönlichen Dämon, in der Illusion einer Außenwelt, aber dadurch, dass sie alle dran teilhaben, zerfällt die letztere nicht in unzählige Privatwelten - man könnte daher von einer Art „Dämonengemeinschaft“" sprechen, welche das menschliche Beziehungsgeflecht durchzieht und aufrechterhält. Der Dämon legt die Maske eines Menschen auf und begegnet einem anderen maskierten Dämon - es handelt sich um ein reines Maskenspiel.

„Wir sind nur Marjonetten, gezogen an fremden uns unbekannten Schnüren. Unser Glük: die Illusion, wir bewegten uns selbst. Wenigstens für den vulgären Menschen. Der Denker weiss genau, wie er dran ist." ${ }^{19}$

In bestimmten Situationen reißt der Schleier der Illusion und es kommt zur Ernüchterung. Dass die Illusion durch eine ,äußere Entlastung“ zerstört wird, will Panizza am Beispiel der geschlechtlichen Liebe aufzeigen. Seiner Ansicht nach geht der geschlechtlich Erregte voll in der Illusion auf, der Gegenstand seines Begehrens sei etwas Besonderes; doch nach dem Geschlechtsakt erlischt allmählich das Interesse an ihm, denn nun wird das eigene Ich in Gestalt des begehrenden Leibs als Quelle der Illusion durchschaut. Mit anderen (desillusionierenden) Worten:

„Während des libidinösen Aktes [erfolgt] die Empfindung des ohnmächtigen Sich-Auflösens in den begehrten Gegenstand, was in dem Moment, in dem das Gehirn die Täuschung verzehrt hat, und die Entlastung erfolgt ist, sich als Wahn ergibt, denn zwei gleich im Begehren, für sich egoistische, stumpfsinnige Leiber bleiben als Resultat zurück." ${ }^{\text {"20 }}$

Panizzas Schrift ist in drei Teile gegliedert: Der erste Teil handelt vom Illusionismus, der zweite vom Dämonismus und, schließlich, der dritte vom Individualismus. Konsequenterweise geht der Illusionismus - die Vorstellung vom illusionären Charakter unseres Realitätsbezugs - in Dämonismus - die Vorstellung, wir werden in unserem Bezug zur Welt von einem (versteckten) Dämon geleitet - über, und dieser entwickelt sich zu einem radikalen Individualismus weiter. Der Individualismus besteht in der Hinnahme des Dämons, seiner Anerkennung, ja geradezu in einem leidenschaftlichen Bekenntnis zu ihm. Die Form, in der der ketzerische Schriftsteller seine Gedanken mitteilt, ist die zweite Form des Personalpronomens, also die Du-Form: Er spricht sich selbst bzw. den Leser direkt an, wodurch seine Gedanken die nötige Pointierung bekommen. Nicht nur dies ist eine Reminiszenz an Max Stirner, der bereits vor Nietzsche den Individualismus in radikalster Form entwickelt hat. ${ }^{21}$ „Mir geht nichts über Mich!“ ${ }^{22}$ ruft der radikale Individualist Stirner aus und fordert einen jeden „Ichträger“" auf, sich von den Hirngespinsten (Illusionen!) zu befreien, mit denen die Gesellschaft die Individualität zu unterdrücken und in letzter Instanz zu negieren sucht. ${ }^{23}$ Ebenso verlangt Panizza vom Individuum, das sich des illusionären Charakters seiner Wirklichkeitsvorstellungen und der Macht seines eigenen Dämons bewusst geworden ist, zu seiner Einzigartigkeit zu stehen und sich selbst voll zu verwirklichen, selbst wenn dies 
zu seinem Untergang innerhalb der „ichfeindlichen“ Gesellschaft führt. Das Individuum ringt sich durch seine Illusionen zur Handlung durch und bestätigt sich dadurch - würde es sich nur in seine eigenen Illusionen verkriechen, so müsste eine bestimmte Geisteskrankheit die unvermeidliche Folge davon sein. Es will aus der Enge der sein Ich (= seinen Dämon) okkupierenden Vorgaben und Vorschriften der Gesellschaft ausbrechen, um sich selbst zu verwirklichen. Panizza nennt historische Persönlichkeiten als konkrete Beispiele, aber auch als Vorbilder für diesen Ausbruch aus den Illusionen in die Tat: Sokrates, Savonarola, Luther. In imperativischer Form lässt der selber vom Dämon getriebene Panizza verkünden:

„Zur Befreiung von Deiner Illusion darfst Du Alles wagen. (...) Deine Seele must Du retten. (...) ,Staat', ,Gesellschaft', ,Religion', ,Ehe', ,Tugendbund', ,Dalai-Lamismus', ,Moral', das sind Illusionen, gegen die Du ankämpfen, und die Du zerstören darfst. Wenn Du’s kanst. Wenn Du musst. Wenn Dich Dein Dämon treibt, Deine letzte Instanz, auf die Du hören musst.“"24

In letzter Instanz ist die Welt selbst das Ergebnis der dämonischen Tätigkeit des Einzelnen, falls er sich zu seinem Dämon bekennt. Daher wird der Dämon als das „Urprinzip des Handelns“ bestimmt. Seine ungewöhnliche Schrift schließt Panizza folgerichtig mit einem kategorischen Imperativ ab:

„Handle, wie Dir Dein Dämon vorschreibt.“25

Wer auf seinen Dämon hört, der verwirklicht sich selbst in der Welt und bringt diesen Dämon zum Ausdruck - selbst auf die Gefahr hin, dabei zugrunde zu gehen. Damit scheint Panizza sein eigenes Ende vorausgesehen zu haben.

\section{Konsequenzen der illusionistischen Weltanschauung}

Was hat es mit dem Gedanken auf sich, die Welt als ein Halluzinationsprodukt zu interpretieren? Werden damit nicht die Grenzen der Normalität überschritten? Aber was heißt hier ,normal'?

18

Ebd., S. 45.

19

Ebd., S. 63. - Sagen das nicht heute auch die „Neurophilosophen“, welche im Denken nur ein Produkt der Gehirntätigkeit sehen und von der Illusion der Willensfreiheit sprechen? Nur sie wollen nicht das mystische Wort, Dämon' in den Mund nehmen. Aber wer weiß, vielleicht sollte man unser Gehirn dämonisch nennen.

20

Ebd., S. 68.

21

Es gibt Autoren, welche Stirner als (geheime) Quelle des Philosophierens Nietzsches angeben. Eduard von Hartmann spitzt diese Vermutung noch weiter zu und meint, dass Nietzsche Stirners Philosophie des Einzigen bloß imitiert hat, ohne ihre Originalität zu erreichen. Vgl. zu dieser „maliziösen Strategie“ Hartmanns Jean-Claude Wolf (Hrsg.), Eduard von Hartmann - Zeitgenosse und Gegenspieler Nietzsches, Königshausen \& Neumann, Würzburg 2006, S. 9 f(Einleitung).

22

Max Stirner, Der Einzige und sein Eigentum, Reclam, durchgesehene und verbesserte Ausgabe, Stuttgart 1981, S. 5.

23

Der eben erwähnte Hartmann deutet Stirners Weltanschauung selbst als ,absoluten Illusionismus“. Vgl. Eduard von Hartmann, „Stirners Verherrlichung des Egoismus“, in: J.-C. Wolf (Hrsg.), Eduard von Hartmann Zeitgenosse und Gegenspieler Nietzsches, S. 69-83, entsprechende Stelle: S. 82. 24

O. Panizza, „Der Illusionismus und die Rettung der Persönlichkeit“, S. 69.

25

Siehe ebd., S. 73. 
Man kann mindestens zwei Lesarten von Panizzas Schrift über den Illusionismus vorschlagen. Die erste wäre eine psychologisch-realistische, die zweite eine ästhetisch-ironistische. Ein Psychologe könnte diese Schrift als Ausgeburt eines kranken Geistes deuten - denn wie soll man anders mit dem Gedanken umgehen, welchem zufolge die Welt bloße Illusion und Projektion eines inneren Dämons wäre, der sich im eigenen Selbst versteckt? Würde man Panizzas Ideen zustimmen, so müsste sich die Grenze zwischen psychischer Gesundheit und psychischer Krankheit auflösen. Wenn der Kranke die Welt qua Illusion richtig sieht (wenn die Wahrnehmung der Welt mit der Illusion zusammenfällt), dann müsste die Möglichkeit entfallen, ihn als krank zu bezeichnen und zu heilen suchen. Nach Panizza sind eher die Maßnahmen, welche die Seelenärzte ergreifen, um angeblich Kranke von ihrer Not zu befreien, unterdrückend und krankmachend. Die Perspektive verkehrt sich somit: Die Kranken sind eigentlich gesund, aber die selbst ernannten Hüter der seelischen Gesundheit machen die Menschen krank, unter anderem durch die Ignorierung der manchmal (wie im Falle der Künstler) idiosynkratischen Individualität, welche vor allem im geistigen Schaffensprozess neue Möglichkeiten der Artikulation erfährt. Daher die Wichtigkeit der Eingebung und der Inspiration, auf die Panizza immer wieder hinweist. Denn in diesen schöpferischen Augenblicken ist man gerade nicht Herr über sich selbst, sondern etwas geschieht mit Einem, das ihn zu einem Anderen macht. Der Mensch verwandelt sich geradezu durch den Akt des Schaffens, der Augenblick der Inspiration kann der Beginn einer neuen intellektuellen oder künstlerischen Entwicklung und Entfaltung des eigenen Selbst werden. Panizza weiß aus eigener Erfahrung, was es mit diesem Prozess der Verwandlung des Selbst durchs Schaffen auf sich hat - deshalb thematisiert er ihn überhaupt.

Man kann den Text aber auch anders lesen - gewissermaßen denselben als doppelbödig verstehen. Vielleicht ist dieser Text auch eine Art Parodie philosophischer Texte, vor allem jener mit dem Anspruch, eine neue Weltanschauung anzubieten. Panizza ist sich möglicherweise der Absurdität einer illusionistischen Position bewusst, nicht nur weil in ihr immanente Widersprüche aufgezeigt werden können, sondern auch deswegen, weil sie sich nicht vollends intersubjektivitätstheoretisch integrieren lässt - ein Problem, vor dem eine jede stark individualistische (um nicht zu sagen: solipsistische) Position steht. (Letztlich scheitert jeder Solipsist am Anspruch, von anderen verstanden werden zu wollen - mindestens in dem (metatheoretischen) Sinne braucht er jemanden (mindestens eine Person!), von dem sein Standpunkt akzeptiert wird. Sonst hebt er seine eigene Position auf - scheitert, wie dies Argumentationstheoretiker zu sagen pflegen, am ,performativen Selbstwiderspruch“). Durch die Überzeichnung dieser Position führt er sie gewissermaßen ad absurdum, denn wie könnten wir im gewöhnlichen Leben wie auf der Ebene des theoretischen Denkens und Sprechens funktionieren, wenn wir die Unterscheidung zwischen Illusion und Realität (zumindest in einem schwachen Sinne) aufheben würden? Der gesamte Diskurs würde in Stücke zerfallen. Es gehört zu den Eigenheiten, ja geradezu Idiosynkrasien des fränkischen Schriftstellers, Extreme auszuschöpfen, buchstäblich: mit Seele und Leib, wie dies auch sein bewegter Lebenslauf gezeigt hat.

Ein Merkmal dieses Autors ist auch seine ironische Haltung, welche vor keiner Autorität, egal ob weltlicher oder kirchlicher, Halt macht. Dass Panizza durchaus zu versteckter Ironie auch bei der Verfassung von theoreti- 
schen Texten tendiert, kann man anhand seiner Schrift Psichopatia criminalis (1898) illustrieren. In ihr schlägt er einen quasi-fachlichen Ausdruck für eine bestimmte Art von Geisteskrankheit vor, welche viel schlimmer als andere psychische Krankheiten sei, da sie zu Ungehorsam und zur Untergrabung von staatlicher Autorität führe, weshalb er den Repräsentanten der Macht dringend rät, diese Krankheit als solche zu erkennen und ihrer Verbreitung durch entsprechende Maßnahmen vorzubeugen. Das eigentlich Schlimme an dieser Krankheit ist, dass sie Merkmale der schwierigsten Geisteskrankheiten, als da wären: Gehirnerweichung (paralisis cerebri), Tobsucht (mania), Melancholie (melancholia) und Verrücktheit (paranoia), in sich vereint. Psichopatia criminalis wird so zum Oberbegriff aller gefährlichen Geisteskrankheiten. Panizza spricht in diesem Zusammenhang von ,,politischen Gehirnkrankheiten“, was klar seine Ironie zum Ausdruck bringt, denn eine solche Art von Krankheit gibt es nicht - was es aber gibt, ist die repressive Gewalt der politischen Mächte, welche sich gegen jeden Anflug von Kritik, Zweifel und Aufstand regt und diese einzudämmen versucht. Die politischen Regime sind eigentlich krank, nicht die unter ihr Joch geworfenen Individuen - so lässt sich prägnant der Sinn von Panizzas Ausführungen zu jener imaginären Krankheit fassen. Sarkastisch bemerkt er:

„Bei der originär sowieso gegebenen verrükter [-en?] Anlage der deutschen unsterblichen Seele (...), müsste es sonderbar zugehen, wenn sich nicht bei einem Professor, bei einem Dozenten, bei einem denkenden Proletarjer, bei einem verbissenen und verbohrten Demokraten, bei einem fingerkauenden Schurnalisten und Schriftsteller, einem freisinnigen Theologen die nötige Porzjon fände, um die Bedingungen für das Zustandekommen der psichopatia criminalis juristisch als gegeben zu erachten, und damit die Überführung in die rettende Staats-Anstalt zu ermöglichen." ${ }^{26}$

Panizza nennt eine Reihe von wichtigen historischen Gestalten, welche seiner diagnostischen Ansicht nach von jener fatalen Krankheit befallen waren und welche für eine psychiatrische Behandlung reif gewesen wären: die Gracchen-Brüder, Savonarola, Luther, Kant, Schiller, Hegel, Feuerbach, Bruno Bauer, Lassalle, Nietzsche u. a. ${ }^{27}$ Am Ende seiner Schrift rät Panizza den Fürsten Europas ihre „heiligsten Güter“ zu wahren, ${ }^{28}$ indem sie die „kriminellen Psychopathen" hinter Gitter bringen oder den Seelenärzten übergeben. ${ }^{29}$ Als ob er dadurch sein eigenes Schicksal vorausgeahnt hat - denn einige Jahre nach dem Erscheinen dieser Schrift wird ihr Autor selbst in die psychiatrische Klinik eingeliefert und damit „unschädlich“ gemacht.

26

Oskar Panizza, „Psichopatia criminalis. Anleitung um die vom Gericht für notwendig erkannten Geisteskrankheiten psichjatrisch zu eruïren und wissenschaftlich festzustellen. Für Ärzte, Laien, Juristen, Vormünder, Verwaltungsbeamte, Minister etc.“, in: O. Panizza, Psichopatia criminalis und andere Schriften, S. 101-139, S. 134.

27

Ein eigenes ,psychopathologisches“ Profil erstellt Panizza auch von Max Stirner, von dessen Denken er besonders angetan war, wie die zu Ehren Stirners geschriebene Illusionismus-Schrift bereits gezeigt hat. Vgl. ebd., S. 134 f.
28

Vgl. ebd., S. 139.

29

Er schlägt gleich auch effektive Heilungsmethoden vor: „Die milde Behandlung, richtig temperierte Wannenbäder, die Ruhe, die Abgeschlossenheit, Nachtigallenschlag jenseits der Gitter, der gütige Zuspruch des Arztes - ein Bischen Hyoscyamin, und ein Bischen Bromkali - und die politische Einsicht all' dieser Internierter wäre bedeutend gewachsen." - Ebd., S. 102. Die beißende Ironie dieser Stelle ist nicht zu übersehen - denn wie soll man durch Einnahme von Medikamenten politische Ansichten therapieren? 


\section{Aporien eines (eingebildeten?) Geisteskranken}

Hat das letztlich tragische Schicksal des Skandalautors Panizza die Gefahren aufgezeigt, welche aus einer illusionistischen Weltanschauung für ihren Vertreter folgen? Hat seine Internierung in ein Sanatorium seine Lebensansicht ad absurdum geführt oder - gerade umgekehrt - ihre Richtigkeit vollends bestätigt? Gehört nicht jeder Illusionist in eine psychiatrische Anstalt? Muss man nicht damit rechnen, von seiner Umgebung für unzurechnungsfähig gehalten zu werden, wenn man nicht mehr Illusion von der Realität zu unterscheiden weiß oder die Wirklichkeit selbst für einen Schein hält? Scheitert man mit dieser Ansicht, wenn nicht am gesunden Menschenverstand, dem wir letztlich beim alltäglichen Umgang mit den Dingen vertrauen, dann doch an den sozial bedingten Rahmenbedingungen unserer Realitätsvorstellungen, welche klar das Reale vom bloß Scheinbaren zu trennen wissen? Bevor man diese Fragen zu beantworten sucht, sollte man sich die viel fundamentalere Frage stellen, ob Panizza nicht gerade diese Rahmenbedingungen, welche die Unterscheidung zwischen Wirklichkeit und Schein, gerade doppelt - durch sein Werk und seine Person - in Frage stellen wollte. Panizza in Person - die Empörung, mit der man seine ketzerischen Schriften quittiert hat, die Härte, mit der man gegen ihn vorgegangen ist - ist das Beispiel dafür, wie bzw. wo man endet, wenn man sich gegen soziale Zwänge auflehnt, welche die Individualität (vor allem der schaffenden Menschen) in ein enges Identitätskorsett hineinpressen und dem Leben der Unangepassten das Störrische wegnehmen möchten. Panizza musste einen hohen Preis für seinen rebellischen Ausbruch aus diesen Zwängen zahlen - sein Beispiel kann als Warnung, aber vielleicht für andere auch als Inspiration und Herausforderung genommen werden, sollten Individualität und Kreativität wieder auf der Tagesordnung des philosophischen Diskurses stehen.

Das, was den Fall Panizza sowohl verwirrend als auch interessant macht, ist eine Art Spiel, in welche dieser Provokateur seine Mitmenschen und Leser einzubeziehen wusste - wenn am Ganzen nicht der Zufall schuld sein sollte. Es stellt sich nämlich die Frage, ob eine geisteskranke Person überhaupt mit den Realitätsschablonen so geschickt umzugehen vermag, wie dies Panizza in manchen seiner Schriften zu tun wusste. Kann sich eine psychisch labile Person so verhalten, als ob sie wirklich krank wäre - oder ist bereits dies, sich als Halluzinant auszugeben, bereits ein Symptom von Geisteskrankheit? Gehört nicht zu einem solchen Spiel mit Normalitätszuschreibungen und Realitätsstereotypen eine gewisse strategische Intelligenz des eingebildeten Kranken, welcher gleich einem geschickten Schachspieler bei jedem Zug weiß, was der Gegner tun wird? Unberechenbarkeit gehört zu Panizzas Charakterzügen die Frage ist nur, ob zu den positiven oder den negativen. Seine Bemühungen, sich in die psychiatrische Einrichtung internieren zu lassen, mögen zwar als Folge realer paranoider Vorstellungen verstanden werden, welche sich während seines Aufenthalts im Ausland (vor allem in Paris nach 1901) unter dem Einfluss realer Gefahr, von den polizeilichen Handlangern des Kaisers Wilhelm II. verhaftet zu werden, entwickelt haben und Panizzas Geist gepeinigt haben - dennoch, es bleibt ein Schimmer von Zweifel, ob auch im scheinbaren Delirium der kontroverse Schriftsteller seine Umwelt nicht ein weiteres Mal schocken wollte (man denke an den Vorfall, als er an jenem Oktobertag des Jahres 1904 nur im Hemd bekleidet durch die Münchener Straßen spazieren ging). Warum wehrt er sich gegen die Entscheidung gegen seinen Willen entmündigt zu werden, wenn ihm das als einem geistig bereits Umnachteten egal 
sein könnte? Seine autobiografische Notiz, vorgetragen im Entmündigungsverfahren vor dem Münchener Amtsgericht am 28. März 1905, ${ }^{30}$ zeugt davon, dass Panizza über die eigenen Gesundheitsprobleme zu reflektieren und sich ein Bild von der eigenen Krankheit durchaus zu machen wusste. Anders als Nietzsche, der nach seinem Zusammenbruch nicht mehr fähig war, sich auf sich selbst zu besinnen, wehrte sich der Dämon in Panizza noch eine Weile, auch wenn in seinem Fall kein positiver Ausgang mehr möglich war. Und am Ende kann man sich dennoch nicht des zweifelnden Eindrucks erwehren, ob der von seinem Dämon besessene Schriftsteller nicht bewusst nach seiner Einlieferung in die psychiatrische Anstalt lechzte, um letztlich dem Wahnsinn der äußeren Welt zu entfliehen.

Panizzas Fall wirft schlussendlich die Frage auf, wo die Grenze zwischen wirklicher und bloß eingebildeter Krankheit verläuft. Die volkstümliche Weisheit, welche selbst bloß eingebildet sein mag, nach der jemand so viel gesund ist, solange er sich gesund fühlt, kann vermutlich nicht ins Gegenteil verkehrt werden, so dass man sagen könnte, dass man in dem Maße an einer Krankheit leidet, in dem man selbst daran glaubt, von ihr betroffen zu sein. Wohlbefinden stärkt das eigene Selbstvertrauen, eingebildetes Missbefinden scheint zu zeigen, dass man das Selbstvertrauen verloren hat - buchstäblich, dass das Verhältnis zum eigenen Selbst gestört ist. Ist letztlich Panizza an seiner Aufmüpfigkeit gegenüber allen Autoritäten, an der eigenen Ego-Stärke, also am eigenen Dämon gescheitert? Mit seinem rebellischen Verhalten hat er zusätzlich den sozialen Druck auf sich verstärkt. Gehört dieser unbändige Wille, ja geradezu die Sucht danach, mit dem eigenen Kopf gegen jene Wand zu stoßen, welche nicht fallen wird, bereits in das Krankenbild Panizzas mit hinein? Lässt sich das Stereotyp des „,grundlosen Rebellen“ auf ihn anwenden? Wenn man sich auch die Gegner einbildet, dann verstärkt man das eigene Krankheitsbild.

Sicherlich ist der Gegner, gegen den sich Panizza in und mit seinen Schriften widersetzte, nicht bloß eingebildet gewesen - er war ebenso real wie die gegen ihn vorgenommenen Maßnahmen (Verbot seiner Schriften, Anklage, der gerichtliche Prozess, die Verurteilung zur Haftstrafe, steckbriefliche Verfolgung, Beschlagnahmung des Vermögens, Internierung in die psychiatrische Anstalt, Entmündigung gegen seinen Willen). Es gehörte zum Wesen des wilhelminischen Reichs als eines konservativen Regimes, jeglichen Anflug von Kritik und Nonkonformismus aufzuspüren und im Keim zu ersticken, ferner diejenigen ketzerischen Denker, die sich gegen es auflehnten, umso strenger zu ahnden und aus dem Verkehr zu ziehen - und im Falle des fränkischen Schriftstellers tat es dies umso mehr, weil sich dieser dagegen ,wie eine Hyäne" ${ }^{\text {311 }}$ wehrte. Man mache sich daher keine Illusionen (!) über Panizzas Lage - auch wenn es so zu sein scheint, dass er sich in philosophische Gedankenspiele über die Auflösung der Grenze zwischen Realität und Illusion flüchtete, sein Leiden war real und sein Dämon entwickelte sich als verständliche Reaktion gegen den Wahnsinn der äußeren (sozialen) Welt, die ihn zu ersticken drohte. Aber selbst wenn seine Gegner real waren, steigerte sich Pa-

30

Vgl. nochmals O. Panizza, ,Selbstbiographie" , a. a. O.

31

Vgl. zu dieser „Kampfansage“ den Auszug aus einem Brief an Max Halbe in: O. Panizza,
„Meine Verteidigung in Sachen ,Das Liebeskonzil"“, a. a. O., S. 4. 
nizzas berechtigte Angst vor der Verfolgung in einen richtigen Verfolgungswahn, der umso stärker auf ihn wirkte, als kein Ausweg aus dieser misslichen Situation gefunden werden konnte. Möglicherweise hat sich Panizza in seine illusionistischen Gedanken so tief verbohrt, dass er am Ende tatsächlich nicht mehr die Wirklichkeit vom Schein zu trennen wusste, sodass die geistige Verwirrung die logische Folge einer solchen Bewusstseinsentfremdung war. Es ist daher kein Zufall, dass sich Panizza mit dem Illusionismus als möglicher philosophischer Weltanschauung auseinandergesetzt hat - denn der Realismus entfiel von vornherein als Weltanschauung der Angepassten. Bei ihm ist der Fall umgekehrt: Der Wirklichkeitsglaube verblödet den schöpferischen Menschen, die eigene Einbildung macht ihn glücklich.

Welches sind nun die Aporien der illusionistischen Weltanschauung Oskar Panizzas? Zunächst einmal zeigt uns sein Fall (im doppelten, psychologischen wie philosophischen Sinne: Verfall der Persönlichkeit und Verlust der „Welthaftigkeit“), welche Probleme derjenige hat, der die Wirklichkeit zur Illusion abstempelt - ihn holt auf anderen Wegen die (soziale) Wirklichkeit ein. Panizza beginnt seine Berufskarriere als Seelenarzt, endet aber dann selber in den Händen von Seelenärzten. Er zeigt am eigenen Beispiel wie die eigene Kreativität zum Verhängnis werden kann, wenn man sich zu sehr einbildet, genial zu sein. ${ }^{32}$ Für die Bioethik ist zweifellos am interessantesten der Zusammenhang von Disziplinarmacht und Normalitätsvorstellungen, welche der „Fall Panizza“ an den Tag legt: Wer die staatliche oder kirchliche Autorität zu untergraben sucht, wird entweder ins Gefängnis oder ins Irrenhaus geschickt - oder gleich beides, wie dies mit Panizza passiert ist. Eine Lektion aus der Geschichte der Disziplinargesellschaft und des Übergangs der Disziplinarmacht vom körpertechnischen auf den seelenanalytischen Vollzugsmodus, wie ihn u. a. Michel Foucault beispielhaft beschrieben hat. ${ }^{33}$ Es würde sich lohnen, eine Studie zum Fall Panizza in machtgenealogischer Manier zu verfassen, mit Nachdruck auf der Darstellung des Kontrasts von repressiven Techniken der Selbstdisziplinierung und produktiven Weisen der Selbstpflege in schriftstellerischen Praktiken. Mit seiner Art, die repressiven Mitteln des Systems herauszufordern, aber auch infolge seiner spezifischen biografischen Situation, welche uns den tragischen Fall zeigt, wie ein Seelenarzt selbst zum Pflegefall wurde, hat der verfemte Provokateur Panizza jenes Unbehagen antizipiert, infolge dessen sich in der Nachkriegszeit zunehmend offener Widerstand gegen den Umgang mit psychisch Kranken in psychiatrischen Anstalten bilden und konkrete Gestalt annehmen wird (Antipsychiatrie). Der „Fall Panizza" dürfte daher für jene Zeitgeistforscher von Wichtigkeit sein, welche den Gründen dieses Unbehagens nachgehen möchten.

Welche tieferen Lehren kann die Philosophie aus dem Fall Panizza, aus den Aporien seiner geschundenen Seele ziehen? Vielleicht sollte man sich gar nicht die Mühe geben, solche Aporien auflösen zu wollen - sie machen gerade den „Fall Panizza“ so spannend. Ein jeder Versuch einer apodiktischen Schlussfolgerung scheint nur einen Teil des Problems zu treffen - und so könnte die Einnahme eines einseitigen Standpunkts zeigen, dass man andere Aspekte desselben Problems aus den Augen verloren hat. Doch selbst wenn eine Art bewusster „Unentschiedenheit“ vonnöten ist, um am ehesten der Komplexität des Problems gerecht zu werden, scheint mir dennoch eine Warnung vor einem allzu radikalen (,dämonischen“) Individualismus nicht abwegig zu sein. Mag sein, dass uns zu viel Realismus spießig macht, aber wenn wir uns zu sehr in unsere Illusionen hineinsteigern, verlieren wir unseren Halt 
auf dem Boden der Tatsachen. Legt man den Grund der Welt ins eigene Ich, stürzt man in die Bodenlosigkeit. Ohne den Widerstand der Welt von außen zu spüren, kann sich das Ich gar nicht entfalten. Und eine Gemeinschaft von Illusionisten könnte kaum aufrechterhalten werden, wenn man nicht die Schranken der eigenen Illusion auf den Anderen hin durchbrechen würde. So gravierend die Realität der Außenwelt die Entfaltung unserer Individualität einzuschränken vermag, sollte man nicht ihren Wert bei der Ausbildung und Stärkung des eigenen Selbst verkennen - denn gerade in dem Wunsch, sich an dieser Welt ,abzuarbeiten“, kann der individuelle Schaffensdrang entfacht werden. Den Rückzug aus der bedrohlichen Außenwelt in seine dämonische Innenwelt musste der Illusionist Oskar Panizza teuer bezahlen. Wenngleich er sich der Ausweglosigkeit der eigenen Mission im Klaren war, wie das vom folgenden Zitat aus seiner philosophischen Schrift belegt wird:

„Handle, wie Dir Dein Dämon vorschreibt. Schreckst Du vor den Konsequenzen in der Welt der Erscheinungen zurük, dann ist sie stärker wie Du. Sezt Du Dich durch, dann bist Du Obsiegender. Du gehst vielleicht zu Grund. Aber zu Grunde zu gehn in der Welt der Erscheinungen, ist ja das Loos von uns Allen. ${ }^{\text {"34 }}$

\title{
Damir Smiljanić
}

\section{»Čini kako Ti nalaže Tvoj demon!«}

\section{Iluzionistički pogled na svijet Oskara Panizze i njegove aporije}

\begin{abstract}
Sažetak
U članku će biti riječi o burnom životu njemačkog pisca, esejista i satiričara Oskara Panizze (1853. - 1921.), kao i o njegovu sukobu sa zakonom i vlašću, zbog čega je morao provesti godinu dana u zatvoru. Balansirajući na granici između stvarnog i iluzornog, genijalnosti i ludila, ovaj karizmatični autor proživio je život pun proturječja: započeo je kao liječnik za mentalne bolesti, postao pisac, a svoju životnu odiseju okončao kao pacijent u sanatoriju. Nadovezujući se na Maxa Stirnera, Panizza je zastupao iluzionizam kao legitiman pogled na svijet: halucinacija je ono stvarno, a stvarnost samo umišljena. U članku će se pokazati da se ne radi o stvaratelju pomračenog uma, već o nekome tko je preispitivao granicu između normalnog $i$
\end{abstract}

32

In seinem Vortrag „Genie und Wahnsinn“, welchen er am 20. März 1891 vor den Mitgliedern der naturalistisch orientierten $\mathrm{Ge}$ sellschaft für modernes Leben gehalten hat, thematisiert Panizza ein während des 19. Jahrhunderts beliebtes Thema: die Beziehung zwischen genialer Begabung und geistiger Krankheit, welche bei vielen bekannten schöpferischen Menschen anzutreffen ist. Zwar sollte man Genialität nicht mit Wahnsinn gleichsetzen (vor allem warnt er vor dem fatalen Rückschluss: Wahnsinn sei Genie) dennoch gibt es gewisse Anzeichen bei schöpferischen Menschen, welche auf eine fragile Gemütsverfassung hinweisen. Panizza nennt deren zwei Formen: Neigung zu Halluzinationen und zur psychischen Epilepsie. Erstere kommt infolge von Sinnestäuschungen auf, letztere zeichnet sich durch Aufhebung des Bewusstseins und visionäre Delirien auf. Nicht zufällig befasst sich der ständig zwischen Schaffensdrang und Hang zu Skandalen pendelnde Schriftsteller mit diesem Thema. Vgl. dazu Oskar Panizza, „Genie und Wahnsinn", in: O. Panizza, Psichopatia criminalis und andere Schriften, a. a. O., S. 74-100, S. $91 \mathrm{ff}$.

33

Vgl. dazu stellvertretend Michel Foucault Überwachen und Strafen. Die Geburt des Gefängnisses, Suhrkamp Verlag, Frankfurt a. M. 1977.

34

Oskar Panizza, „Der Illusionismus und die Rettung der Persönlichkeit“, S. 73. 
nenormalnog, u izvjesnom smislu anticipirajući nadolazeću kritiku normativnih postavki institucionalne psihijatrije.

\title{
Ključne riječi
}

Oskar Panizza, illuzionizam, demonizam, individualizam, genijalnost, ludilo, Psichopatia criminalis

\section{Damir Smiljanić}

\section{“Do It Like Your Demon Obliges You To Do!"}

\section{The Illusionistic Worldview of Oskar Panizza and Its Aporias}

\begin{abstract}
The article is dealing with the turbulent life of German writer, essayist and satirist Oskar Panizza (1853-1921), also with his attacks against the law and authorities, because of which he was sentenced to one-year imprisonment. Balancing on the boundary line between reality and illusion, ingeniousness and insanity, this charismatic author spent a life full of contradictions: he began to work as a neurologist, but shortly afterwards he became a writer-finally, he ended his life odyssey as a patient in a sanatorium. Following the ideas of Max Stirner, Panizza subscribed to illusionism as a valid weltanschauung: hallucination is real, the reality is chimerical. The article aims to show that we do not have a case of a mentally disordered person, instead, of an author who was questioning the boundaries between normality and abnormality, anticipating the subsequent criticism of normative assumptions of the institutionalized psychiatry.
\end{abstract}

Keywords

Oskar Panizza, illusionism, demonism, individualism, genius, madness, Psichopatia criminalis

\section{Damir Smiljanić}

\section{«Conforme-toi à ce que te souffle ton Démon !»}

\section{Regard illusionniste sur le monde d'Oskar Panizza et ses apories}

\section{Résumé}

Il sera question dans cet article de la vie tumultueuse d'Oskar Panizza (1853-1921), écrivain allemand, essayiste et satiriste, et également de ses démêlés avec la loi et les autorités, en raison de quoi il a dî passer une année en prison. Se situant à la limite entre le réel et l'illusoire, le génie et la folie, cet auteur charismatique a vécu une vie pleine de contradictions : il a débuté en tant que médecin spécialiste des maladies mentales, est devenu écrivain, et l'odyssée de sa vie a pris fin dans un hopital psychiatrique. En s'appuyant sur Max Stirner, Panizza a défendu l'illusionnisme comme regard légitime sur le monde : l'hallucination est le vrai, et la réalité n'est qu'imaginaire. Cet article montrera qu'il n'est pas question d'un créateur dont l'esprit était obscurci, mais de quelqu'un qui interrogeait la limite entre le normal et l'anormal, qui en un certain sens, anticipait la critique à venir du cadre normatif des institutions psychiatriques.

\section{Mots-clés}

Oskar Panizza, illusionnisme, démonisme, individualisme, génie, folie, Psichopatia criminalis 